\title{
Facility-based surveillance for influenza and respiratory syncytial virus in rural Zambia
}

\author{
Gideon Loevinsohn ${ }^{1,2}$, Mutinta Hamahuwa ${ }^{3}$, Pamela Sinywimaanzi ${ }^{3}$, Katherine Z. J. Fenstermacher ${ }^{4}$, \\ Kathryn Shaw-Saliba ${ }^{4}$, Andrew Pekosz ${ }^{4,5}$, Mwaka Monze ${ }^{6}$, Richard E. Rothman ${ }^{4}$, Edgar Simulundu', \\ Philip E. Thuma ${ }^{3,5}$ and Catherine G. Sutcliffe ${ }^{1^{*}}$
}

\begin{abstract}
Background: While southern Africa experiences among the highest mortality rates from respiratory infections, the burden of influenza and respiratory syncytial virus (RSV) in rural areas is poorly understood.

Methods: We implemented facility-based surveillance in Macha, Zambia. Outpatients and inpatients presenting with influenza-like illness (ILI) underwent testing for influenza A, influenza B, and RSV and were prospectively followed for 3 to 5 weeks to assess clinical course. Log-binomial models assessed correlates of infection and clinical severity.

Results: Between December 2018 and December 2019, 17\% of all outpatients presented with ILI and 16\% of inpatients were admitted with an acute respiratory complaint. Influenza viruses and RSV were detected in 17\% and 11\% of outpatient participants with ILI, and $23 \%$ and $16 \%$ of inpatient participants with ILI, respectively. Influenza (July-September) and RSV (January-April) prevalence peaks were temporally distinct. RSV (relative risk [RR]: 1.78; 95\% confidence interval [CI] 1.51-2.11), but not influenza, infection was associated with severe disease among patients with ILI. Underweight patients with ILI were more likely to be infected with influenza A (prevalence ratio [PR]: 1.72; $95 \% \mathrm{Cl}$ 1.04-2.87) and to have severe influenza A infections (RR: 2.49; 95\% Cl 1.57-3.93).
\end{abstract}

Conclusions: Populations in rural Zambia bear a sizeable burden of viral respiratory infections and severe disease. The epidemiology of infections in this rural area differs from that reported from urban areas in Zambia.

Keywords: Influenza, RSV, Southern Africa, Rural, Risk factors, Severity

\section{Background}

Influenza viruses and respiratory syncytial virus (RSV) are leading causes of global respiratory morbidity and mortality $[1,2]$. In southern Africa their burden is becoming increasingly evident, with each nascent surveillance effort uncovering a considerable prevalence of disease [3, 4]. Available evidence suggests that southern Africa experiences among the highest worldwide influenza- and RSV-related mortality rates $[2,5]$. In South

\footnotetext{
*Correspondence: csutcli1@jhu.edu

${ }^{1}$ Department of Epidemiology, Johns Hopkins University Bloomberg School of Public Health, 615 N. Wolfe Street, Room E6535, Baltimore, MD 21205, USA

Full list of author information is available at the end of the article
}

Africa, for example, influenza-related mortality among elderly adults was found to be over three-fold higher than in the United States [6]. Prevalent malnutrition, HIV infection, and tuberculosis increase the severity of viral illness and may contribute to mortality [7-9]. However, much remains unknown about viral epidemiology and risk factors for severe disease. The overwhelming majority of surveillance and research efforts in southern Africa have been based in urban centers. Information from rural areas is particularly scant, yet this is where the majority of the region's population lives [10]. Rural environments present distinct risk factors for transmission and pathogenesis given lower population density, reduced accessto-care, and higher prevalence of undernutrition and 
extreme poverty [11]. Urban-rural differences in epidemiology and severity have been previously described in sub-Saharan Africa for many infectious diseases including tuberculosis [12], HIV [13], and malaria [14].

In Zambia, published studies on influenza and RSV have been limited to the urban capital of Lusaka and government-led influenza surveillance has been confined to Lusaka and the city of Ndola [4, 15-17]. There are no disease estimates from rural areas and few estimates overall among adult populations $[15,18]$. In the absence of context-specific disease estimates for rural areas, clinicians and public health practitioners have little insight into respiratory disease etiology and risk factors for severe illness, likely contributing to antibiotic overuse and misallocation of scarce clinical resources.

In December 2018, facility-based surveillance for influenza and RSV was established in rural Zambia to evaluate their role in causing respiratory illness and begin to situate rural Zambia in the landscape of regional and global virus transmission. The objective of this analysis was to describe the burden of influenza and RSV disease during the first year of surveillance and explore predictors of severe disease.

\section{Methods}

\section{Study site and population}

Facility-based surveillance was established at Macha Hospital in Southern Province, Zambia as part of the Johns Hopkins Center for Excellence in Influenza Research and Surveillance (JHCEIRS) with the primary goal of understanding the burden and epidemiology of influenza virus in this area. Macha Hospital is a 208-bed, district-level hospital that serves a catchment population of approximately 150,000, predominantly subsistence farmers. Southern Zambia historically experiences three seasons: a single rainy season from November to April, a cool dry season from May to August, and a warm dry season from September to November [19]. Malaria prevalence in the area has steadily declined and was $<1 \%$ in recent years [20]. Provincial vaccination coverage for Streptococcus pneumoniae and Hemophilus influenzae type b among children 12-23 months of age is estimated at $91 \%$ for the full complement of vaccine doses [21]. Influenza vaccines and antiviral medications are unavailable in the study area.

\section{Surveillance procedures}

Beginning on December 10, 2018, all outpatients presenting for care to the outpatient department (OPD) and patients newly admitted to the adult and pediatric wards with respiratory symptoms were screened for influenzalike illness (ILI). ILI was defined based on the Centers for Disease Control and Prevention (CDC) definition as measured $\left(\geq 38{ }^{\circ} \mathrm{C}\right)$ or reported fever with either cough or sore throat, either with clinical onset and/or worsening within 7 days of hospital presentation [22]. Reported fever was added to the CDC case definition to increase sensitivity and to account for prior antipyretic use. All inpatients and an age-stratified weekly sample of outpatients with ILI were eligible for enrollment (Additional file 1). Enrollment days and times in the OPD were varied to capture the breadth of the outpatient population.

At enrollment, a nasopharyngeal specimen was collected using a flocked swab, placed in universal transport media (Cepheid Inc., Sunnyvale, CA), and stored at $4{ }^{\circ} \mathrm{C}$. Participants were administered a questionnaire by trained study staff detailing sociodemographic characteristics, symptoms, medical history, and potential exposures. Food insecurity was assessed using the Household Hunger Scale, a three-question scale validated for crosscultural use [23]. HIV serostatus, assessed through routine hospital screening, was recorded. Weight, height, respiratory rate, and peripheral capillary oxygen saturation $\left(\% \mathrm{SpO}_{2}\right.$; measured using a handheld pulse oximeter [CMI Health Inc., Alpharetta, GA]) were captured at enrollment. Treatment administered and patient disposition were obtained from the medical record.

Study participants were followed three to five weeks after enrollment to ascertain clinical course. Information on vital status, symptoms, and hospital admissions were obtained via self-report and by reviewing the medical record. For participants who could not be reached in person, the information was collected by telephonic interview.

\section{Laboratory procedures}

Nasopharyngeal specimens were transported to the Clinical Research Laboratory of the Macha Research Trust within one hour of collection. The specimens were tested for influenza A/B viruses and RSV using the Cepheid Xpert Xpress Flu/RSV assay (Cepheid Inc., Sunnyvale, CA) on the day of collection. The Xpert Xpress Flu/ RSV assay has demonstrated a sensitivity/specificity of 98.6\%/99.3\% and $97.9 \% / 99.4 \%$ for detection of influenza A and B viruses, respectively, and $98.1 \% / 99.4 \%$ for RSV when compared with gold-standard laboratory-based RT-PCR assays [24].

\section{Temperature and precipitation}

Temperature and precipitation were captured once per hour using an outdoor HOBO Micro Station weather sensor (Onset Computer Corporation, Bourne, MA).

\section{Statistical analyses}

This analysis reports on the results of surveillance carried out from December 10, 2018 to December 9, 2019. 
Characteristics of the study population were summarized at enrollment. Participants without a test result for influenza virus or RSV were excluded from the analysis $(n=2$; due to death and withdrawal, respectively). Monthly prevalence of influenza virus and RSV infection among all outpatients was calculated using direct standardization based on the age distribution and age-specific ILI prevalence in the outpatient population, and the age-specific prevalence of influenza virus and RSV among those with ILI enrolled in the study. As all inpatients were not systematically screened for ILI, the monthly prevalence of influenza virus and RSV infection was only estimated among inpatients with ILI, and was calculated directly as the proportion of participants with influenza virus and RSV infection. For both patient populations, trends in respiratory illness (ILI for outpatients and acute respiratory infection for inpatients), influenza virus, and RSV prevalence over time were graphically summarized with locally weighted scatterplot smoothing (LOWESS) techniques.

Differences in presenting characteristics between groups defined by viral testing results were assessed among both outpatient and inpatient participants with univariable and age-adjusted log-binomial models. Where the models failed to converge, Poisson regression with robust variance estimation was employed. Separate models were fit for the outcomes of influenza A virus, influenza B virus, and RSV infection respectively. Sociodemographic characteristics, medical history, and presenting symptoms were evaluated as predictors of viral infection. Moderate or severe hunger was defined as a value of two or greater on the Household Hunger Scale [23]. For children 18 years or younger, underweight was defined as a body mass index (BMI) more than two standard deviations below the age- and sex-specific mean using the WHO's Child Growth Standards [25]. For adults, underweight was defined as a BMI less than 18.5. Tachypnea was defined based on age-specific cutoffs [26]. To evaluate factors associated with severe clinical illness among all participants testing positive for influenza virus or RSV infection, age-adjusted log-binomial models were fit with the outcome of severe clinical illness (vs nonsevere clinical illness) separately for influenza A virus, influenza B virus, and RSV. Severe clinical illness was defined as a composite outcome encompassing at least one of: death while under follow-up, respiratory illness requiring hospital admission at enrollment or during follow-up, or peripheral oxygen saturation $\left(\mathrm{SpO}_{2}\right) \leq 92 \%$ at enrollment [27, 28]. Participants with missing information for one or more of these outcomes $(n=114$ for death and hospital admission during follow-up, $\mathrm{n}=134$ for $\mathrm{SpO}_{2}$ ) who were not already designated as severe based on available data were excluded from the analysis ( $\mathrm{n}=168,25 \%$ of participants).

Statistical analyses were performed using STATA version 14 (Statacorp, College Station, TX).

\section{Results}

Influenza-like illness

Between December 10, 2018 and December 9, 2019, 3,677 of $21,492(17 \%)$ outpatients presenting to the Macha Hospital OPD met criteria for ILI. The highest prevalence was observed among patients under 1 year of age and 1-4 years (0-11 months: 712/1615 [44\%]; 1-4 years: $1264 / 2885$ [44\%]; 5-15 years: 536/2586 [21\%]; 16-50 years: $898 / 11,187$ [8\%]; $51+$ years: $240 / 3098$ $[8 \%])$. ILI was common throughout the year, with peaks in prevalence observed from February-April and JulyAugust (Fig. 1A). In age-stratified analysis, the first ILI peak (February-April) was observed only in children under 15 years, while the second peak (July-August) was observed across age-groups (Additional file 3).

During the surveillance period, 279 of 1781 patients (16\%) were admitted with an acute respiratory complaint. Of those screened for enrollment, 166 met criteria for ILI and inclusion in the study. Similar trends over time in the prevalence of acute respiratory infections were found among hospitalized patients as in the outpatient population (Fig. 2).

\section{Influenza viruses and RSV}

Among outpatients with ILI, 650 eligible patients (17\% of outpatients with ILI) were approached for enrollment: 74 (11\%) declined participation and 576 (89\%) were enrolled in the study (Table 1). In addition, 166 eligible inpatients were approached: 70 declined participation (42\%) and 95 (58\%) were enrolled. The most common reason for declining was the absence of family decision-makers (42\% of refusals). There were no significant differences in age $(\mathrm{p}=0.33)$ or sex $(\mathrm{p}=0.58)$ between those declining participation and those enrolled. The age distribution of participants closely mirrored the distribution of respiratory illness in the patient populations (Additional files 4 and 5).

Among outpatient participants, 66 (11\%) were found to be infected with influenza A virus, 34 (6\%) with influenza $\mathrm{B}$ virus, and 61 (11\%) with RSV. The estimated prevalence among all outpatients presenting for care, regardless of symptoms, was $2.06 \%$ for influenza A virus (95\% confidence interval [CI] 1.59\%-2.54\%), 1.09\% for influenza B virus (95\% CI $0.73 \%-1.45 \%$ ), and $1.80 \%$ for RSV (95\% CI $1.38 \%-2.20 \%)$. Among all outpatients presenting with ILI, the estimated prevalence was $12.07 \%$ (95\% CI 9.28\%-14.85\%) for influenza A virus, 6.39\% (95\% CI 


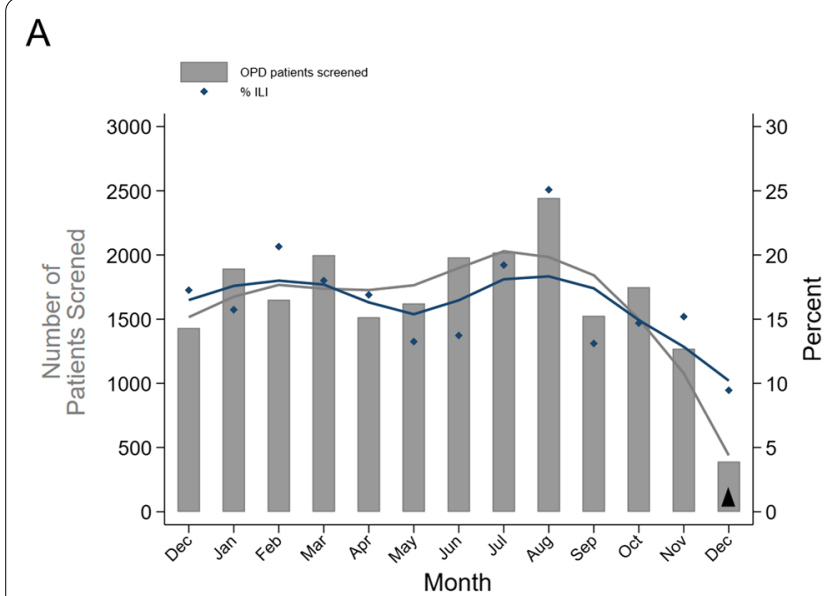

B

C
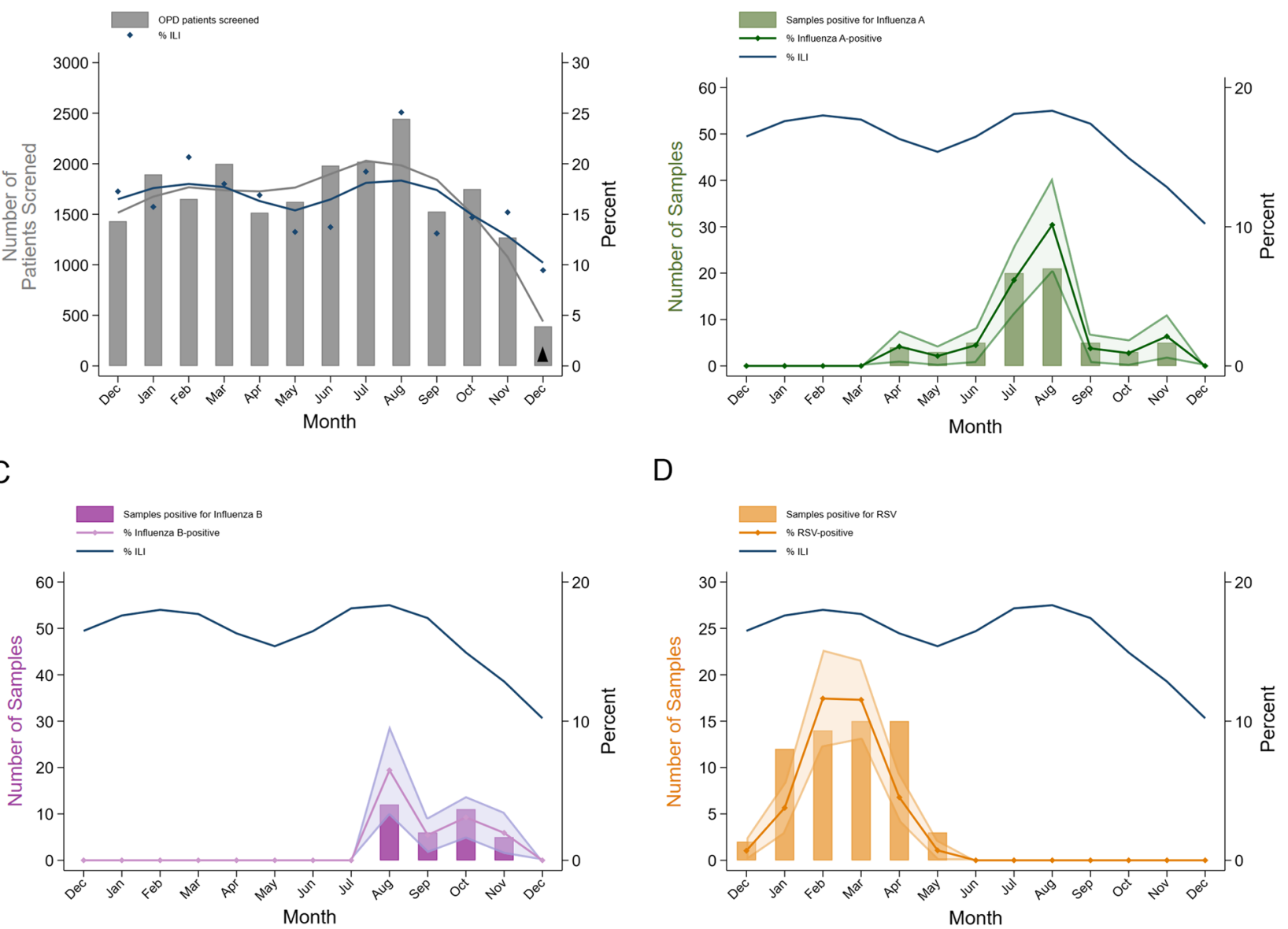

D

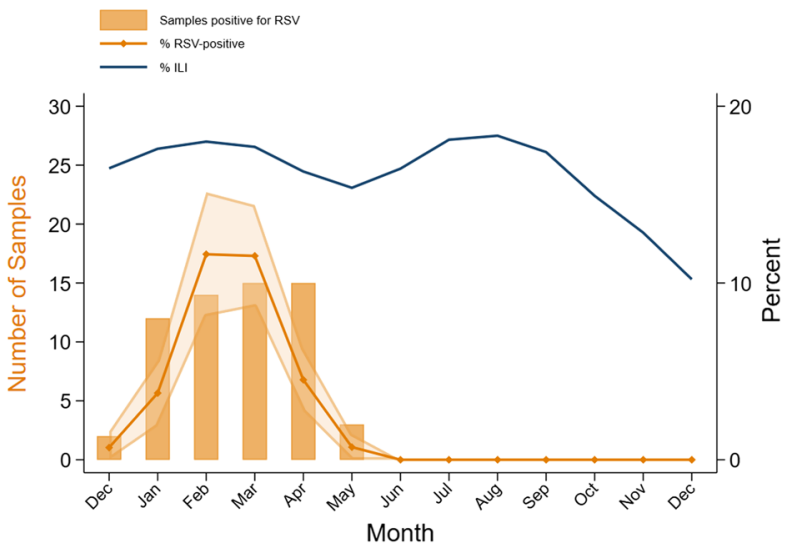

Fig. 1 Trends in (A) ILI, B influenza A virus, C influenza B virus, and D RSV prevalence among outpatients. ILI: influenza-like illness; OPD: outpatient department; RSV: respiratory syncytial virus. Black up pointing triangle: Partial month of surveillance. A OPD attendance and proportion of OPD patients with ILI at Macha Hospital. Gray and blue lines are LOWESS curves for number of patients screened and proportion with ILI, respectively. B-D Viral positivity over the study period for influenza A, influenza B, and RSV, respectively. Bar graph represents the number of positive tests among OPD study participants. Line represents estimated prevalence among all OPD patients with 95\% confidence interval. Blue line represents estimated monthly ILI prevalence among all OPD patients (LOWESS)

4.29\%-8.49\%) for influenza B virus, and $10.51 \%$ (95\% CI 8.04\%-12.98\%) for RSV.

Among all outpatients, distinct temporal trends were observed for both influenza virus and RSV infections during the surveillance period (Fig. 1B-D). RSV infections were detected from December 2018 to May 2019. While this coincides with the historical rainy season, the study coincided with a period of regional drought (Additional file 6). In contrast, influenza viruses were detected during the cold-dry and warm-dry seasons. Influenza A virus was detected from April to November 2019 with the highest prevalence, an estimated $10.13 \%$ of all outpatients (95\% CI 6.71\%-13.56\%), in August. Influenza B virus was detected from August to November 2019, with the highest prevalence, an estimated $6.47 \%$ of all outpatients $(95 \%$
CI 3.22\%-9.72\%), also in August. The two peaks in ILI prevalence coincided with the peaks in RSV and influenza virus prevalence (Fig. 1B-D).

Among inpatient participants with ILI, 20 (21\%) were found to be infected with influenza A virus, 2 (2\%) with influenza B virus, and 15 (16\%) with RSV. Similar temporal trends were observed as for the outpatient population (Fig. 2) with RSV peaking early in the year followed by a peak in influenza virus prevalence later in the dry seasons.

\section{Symptomatology of influenza virus and RSV infections among patients with ILI}

Participants (outpatient and inpatient) with ILI presented to Macha Hospital a median of 3 days after 
A

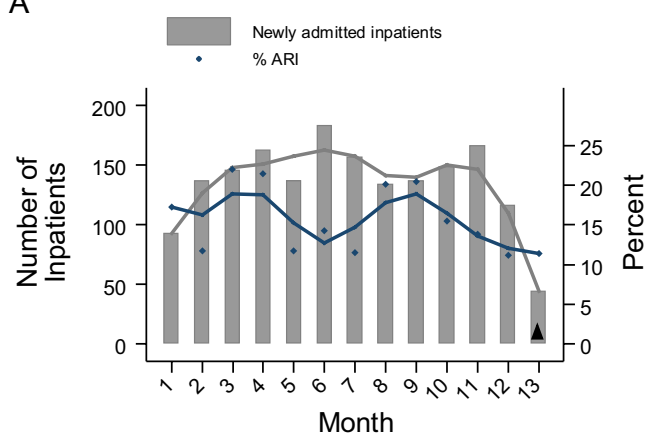

C

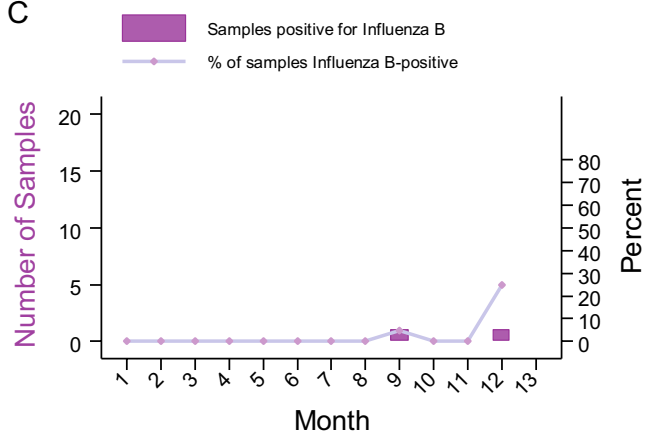

B

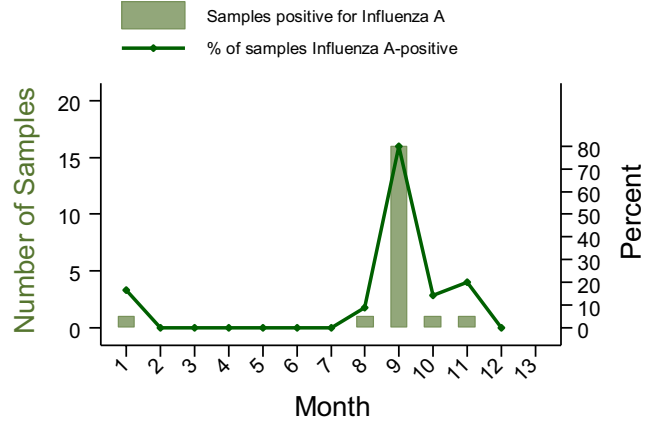

D

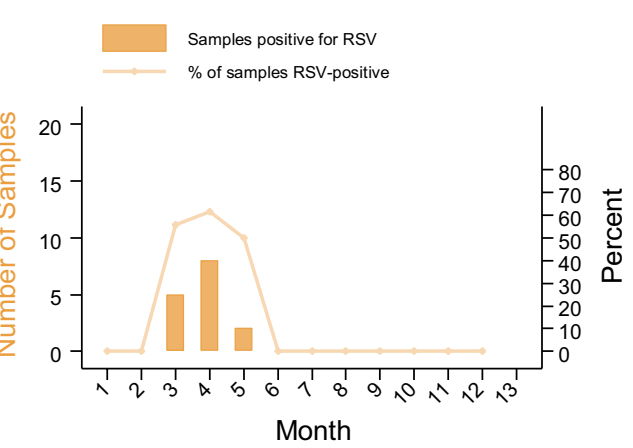

Fig. 2 Trends in (A) ARI among all newly admitted patients, and B influenza A virus, C influenza B virus, and D RSV prevalence among hospitalized patients with ILI. ARI: acute respiratory illness; ILI: influenza-like illness; RSV: respiratory syncytial virus. Black up pointing triangle: Partial month of surveillance. A Newly admitted patients and proportion of all inpatients with ARI at Macha Hospital. Gray and blue lines are LOWESS curves for the number of newly admitted patients and proportion with ARI, respectively. B-D Viral positivity over the study period for influenza A, influenza B, and RSV, respectively, among inpatients with ILI. Bar graph and line represent the number and proportion of positive tests among inpatient study participants with ILI, respectively

symptom onset (IQR 3-4; Additional file 2). There were no significant differences in the duration of symptoms by viral test result. Among children younger than 5 years of age, measured fever $\left(\geq 38{ }^{\circ} \mathrm{C}\right)$ was significantly associated with influenza A virus infection (Prevalence ratio [PR]: 2.29; 95\% CI 1.34-3.91) but not influenza B virus or RSV infection. Those presenting with headache were significantly more likely to be infected with influenza B virus (PR: 9.78; 95\% CI 1.06-90.18) and those presenting with diarrhea were significantly less likely to be infected with RSV (PR: 0.50; 95\% CI 0.27-0.92). The presence of hypoxemia $\left(\mathrm{SpO}_{2} \leq 92 \%\right)$ was significantly associated with RSV infection (PR: 3.10; 95\% CI 1.75-5.49). Among participants older than 5 years, measured fever was significantly associated with influenza A virus (PR: 2.28; 95\% CI 1.19-4.34) and influenza B virus (PR: 7.62; 95\% CI 3.01-19.26) infection, but not RSV. In addition, the presence of tachypnea was significantly associated with influenza A virus infection (PR: 2.90; 95\% CI 1.32-6.39).

\section{Risk factors for influenza virus and RSV infection among patients with ILI}

Inpatient participants were significantly more likely to be infected with influenza A virus than outpatient participants (age-adjusted [adj] PR 1.86; 95\% CI 1.19-2.89; Table 2). There were no significant differences in influenza B virus or RSV prevalence by inpatient status. Influenza $A$ and influenza $B$ viruses were detected across age groups (Table 2, Additional file 3). In contrast, RSV infections were concentrated among children younger than 5 years of age.

With regard to household exposures, the presence of an individual with respiratory symptoms in the household was associated with both influenza A virus (adjPR: 1.54; 95\% CI 1.02-2.31; Table 2) and RSV infection (adjPR: 1.57; 95\% CI 1.02-2.43). Indoor cooking was associated with RSV infection (adjPR: 2.00; 95\% CI 1.12-3.56) but not influenza A or B virus infection. There were few differences in the prevalence of viral infection between those with and without medical comorbidities. However, participants who were underweight were more likely to 
Table 1 Characteristics of the study population at enrollment in Macha, Zambia

\begin{tabular}{|c|c|c|c|}
\hline & $\begin{array}{l}\text { Outpatients } \\
n=576\end{array}$ & $\begin{array}{l}\text { Inpatients } \\
\mathrm{n}=95\end{array}$ & $\begin{array}{l}\text { Overall } \\
n=671\end{array}$ \\
\hline Age in years, median (IQR) & $3.2(0.8-19.0)$ & $2.9(0.8-29.0)$ & $3.2(0.8-19.0)$ \\
\hline $0-5$ months & $61(11 \%)$ & $11(12 \%)$ & $72(11 \%)$ \\
\hline $6-11$ months & $100(17 \%)$ & $15(16 \%)$ & $115(17 \%)$ \\
\hline $1-4$ years & $181(31 \%)$ & $31(33 \%)$ & $212(32 \%)$ \\
\hline $5-15$ years & $77(13 \%)$ & $9(9 \%)$ & $86(13 \%)$ \\
\hline $16-50$ years & $91(16 \%)$ & $18(19 \%)$ & $109(16 \%)$ \\
\hline $51+$ years & $66(11 \%)$ & $11(12 \%)$ & $77(11 \%)$ \\
\hline Female, n (\%) & $316(55 \%)$ & $44(46 \%)$ & $360(54 \%)$ \\
\hline \multicolumn{4}{|l|}{ Educational attainment among adults ${ }^{\mathrm{a}}, \mathrm{n}(\%)$} \\
\hline No education & $11(7 \%)$ & $1(4 \%)$ & $12(7 \%)$ \\
\hline Primary & $101(68 \%)$ & $20(77 \%)$ & $121(69 \%)$ \\
\hline Secondary & $34(23 \%)$ & $2(8 \%)$ & $36(21 \%)$ \\
\hline Post-secondary & $3(2 \%)$ & $3(12 \%)$ & $6(3 \%)$ \\
\hline Travel time (hours) to hospital, median (IQR) & $1.0(0.8-2.0)$ & $2.0(1.0-3.0)$ & $1.0(0.8-2.0)$ \\
\hline Number of individuals in household, median (IQR) & $6(5-9)$ & $7(5-11)$ & $7(5-9)$ \\
\hline Number of individuals sharing sleeping space, median (IQR) & $3(2-3)$ & $3(2-3)$ & $3(2-3)$ \\
\hline Presence of animals in residence compound, n (\%) & $528(92 \%)$ & $84(88 \%)$ & $612(91 \%)$ \\
\hline Food insecurity, n (\%) & $14(2 \%)$ & $6(6 \%)$ & $20(3 \%)$ \\
\hline Underweight, n (\%) & $47(8 \%)$ & $21(22 \%)$ & $68(10 \%)$ \\
\hline HIV-infected, n (\%) & $19(3 \%)$ & $10(11 \%)$ & $29(4 \%)$ \\
\hline History of tuberculosis, n (\%) & $16(3 \%)$ & $6(6 \%)$ & $22(3 \%)$ \\
\hline Number of outpatient medical encounters in past year, median (IQR) ${ }^{b}$ & $1(0-3)$ & $1(0-2)$ & $1(0-3)$ \\
\hline
\end{tabular}

${ }^{a}$ Limited to participants 18 years or older ${ }^{b}$ Excluding the enrollment visit

be infected with influenza A than participants who were not underweight (adjPR: 1.72; 95\% CI 1.04-2.87).

\section{Treatment, outcomes, and clinical severity among patients with ILI}

At the time of presentation, participants with influenza A virus infection were more likely to be diagnosed with sepsis than participants without influenza A (adjPR: 3.50; 95\% CI 1.42-8.62), participants with influenza B were more likely to be diagnosed with a respiratory tract infection than participants without influenza B (adjPR: 1.20; 95\% CI 1.03-1.39), and participants with RSV were more likely to be diagnosed with pneumonia than participants without RSV (adjPR: 3.21; 95\% CI 1.59-6.48; Table 3). Of note, $75 \%$ of all participants presenting with ILI, including $87 \%$ of participants with either influenza virus or RSV infection were prescribed antibiotics. The likelihood of antibiotic prescription was higher for participants with influenza A virus (adjPR: 1.12; 95\% CI 1.02-1.25) or RSV (adjPR: 1.20; 95\% CI 1.10-1.31) than those uninfected.

Overall, clinical severity was assessed in 503 participants (75\% of all participants) and 33\% (165/503) experienced a severe clinical illness. Ten participants died during follow-up, none of whom were infected with influenza or RSV. Participants with RSV were more likely to have a severe clinical illness than those without RSV (age-adjusted risk ratio [adjRR]: 1.78; 95\% CI 1.51-2.11; Table 3), with an increased risk of both hypoxemia (adjPR: 2.62; 95\% CI 1.68-4.06) and hospital admission (adjRR: 1.53; 95\% CI 1.20-1.95). Overall, no differences were observed in the likelihood of severe clinical illness by influenza virus testing result. Among participants with influenza A virus, being underweight was associated with increased risk of severe illness (adjRR: 2.49; 95\% CI 1.57-3.93; Table 4) due to heightened risk of both hospitalization (adjRR: 2.16; 95\% CI 1.03-4.52) and hypoxemia (adjPR: 2.96; 95\% CI 1.19-7.33). We observed no relationship between HIV infection or prior tuberculosis infection and clinical severity. All participants reporting a diagnosis of HIV also reported receiving combination antiretroviral therapy.

\section{Discussion}

We report on the findings from 1 year of facility-based surveillance for influenza and RSV in rural Zambia from December 2018 to December 2019. This is among the first efforts to characterize the burden of viral respiratory disease in a rural southern African setting. At our study 


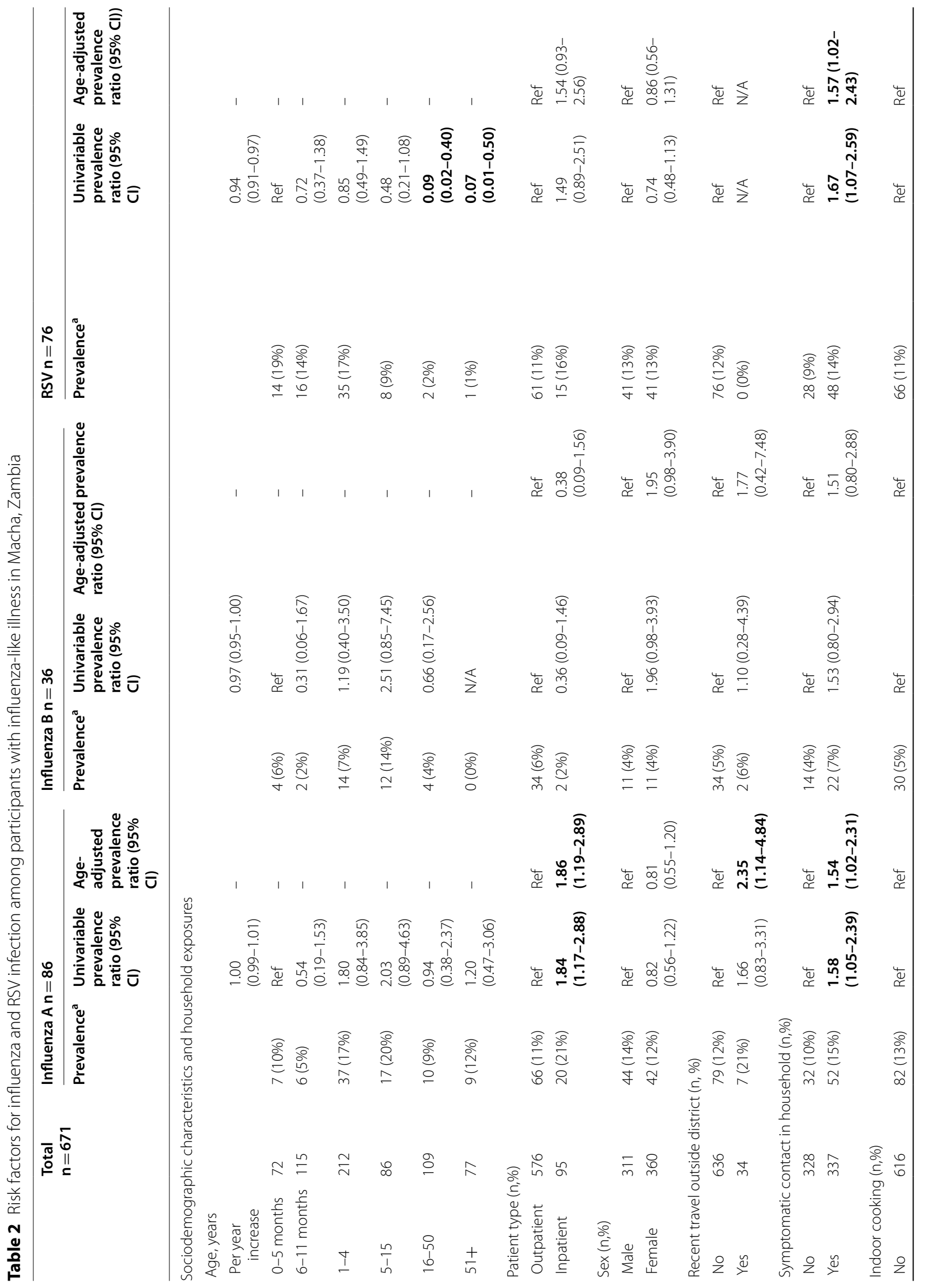




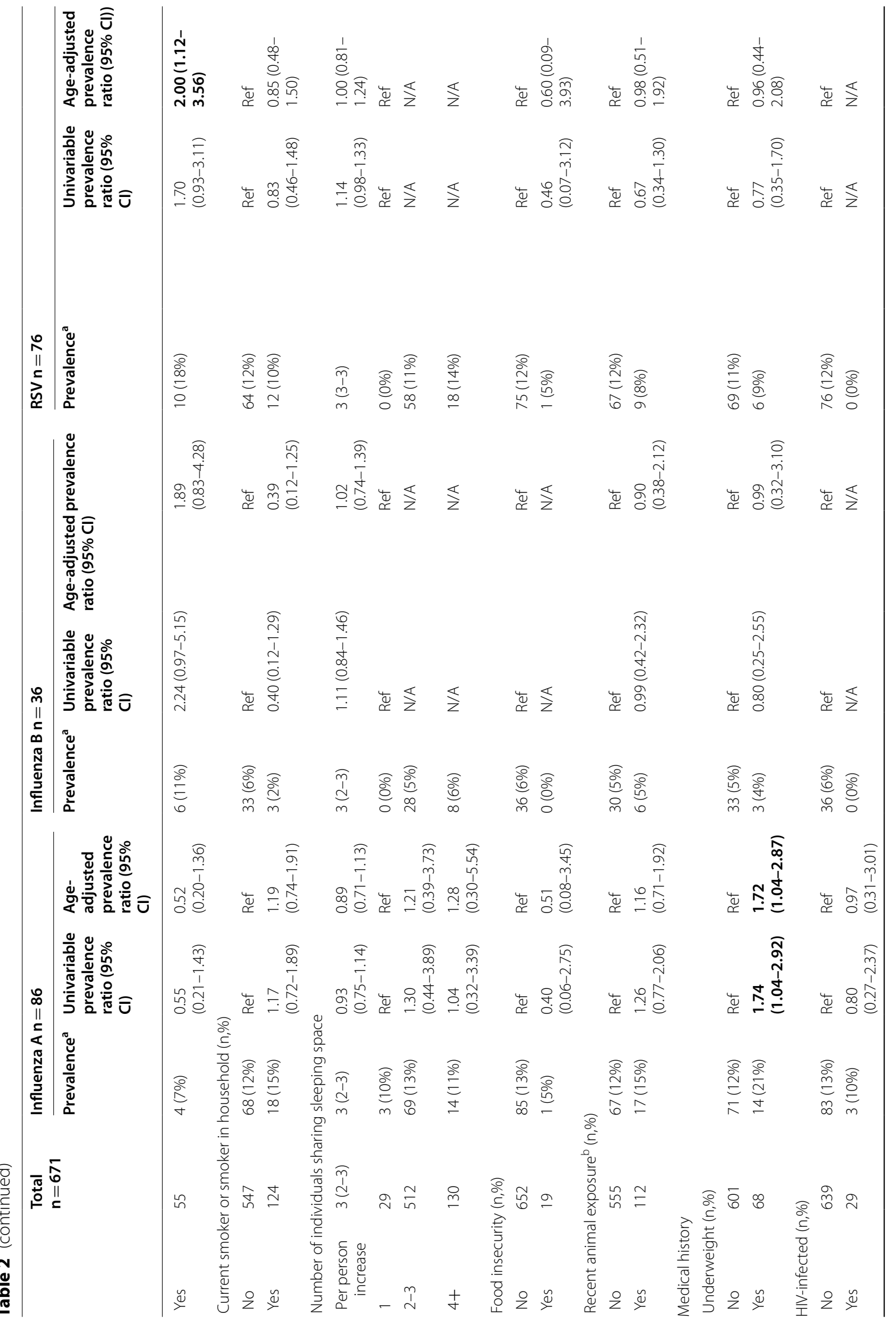




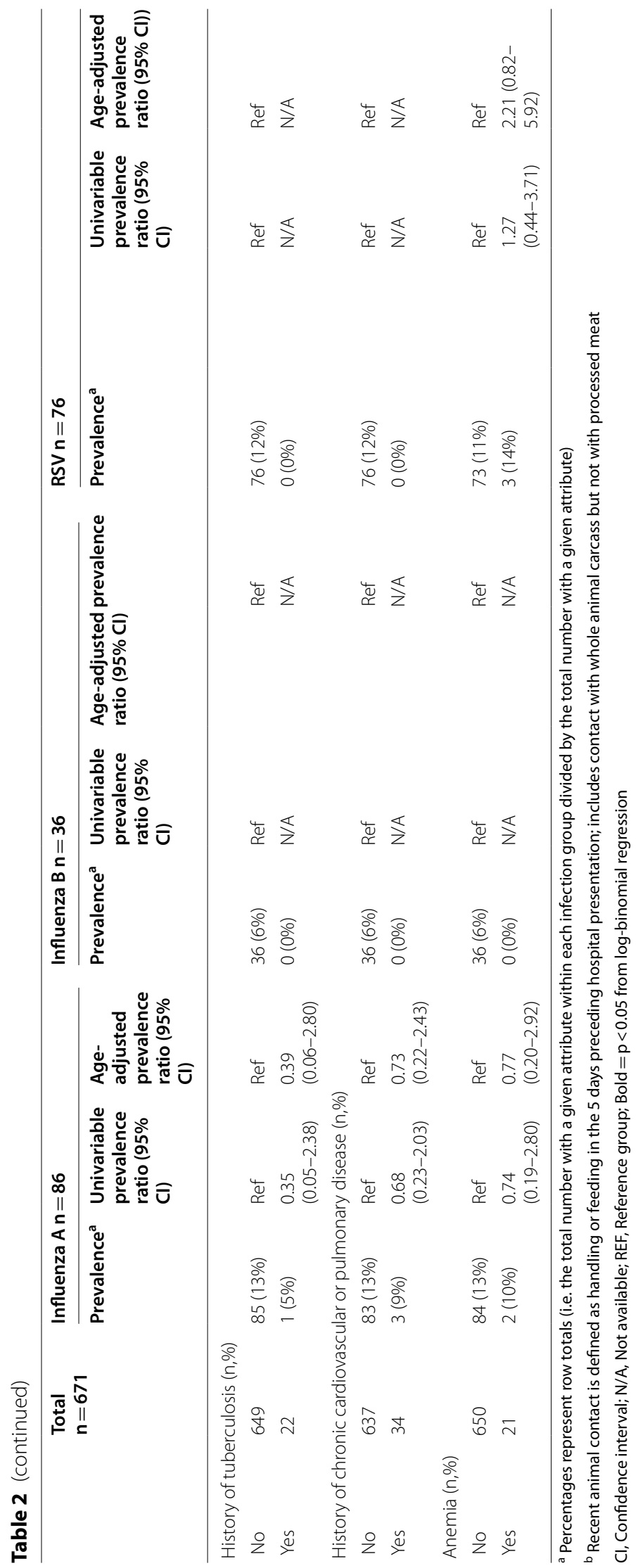




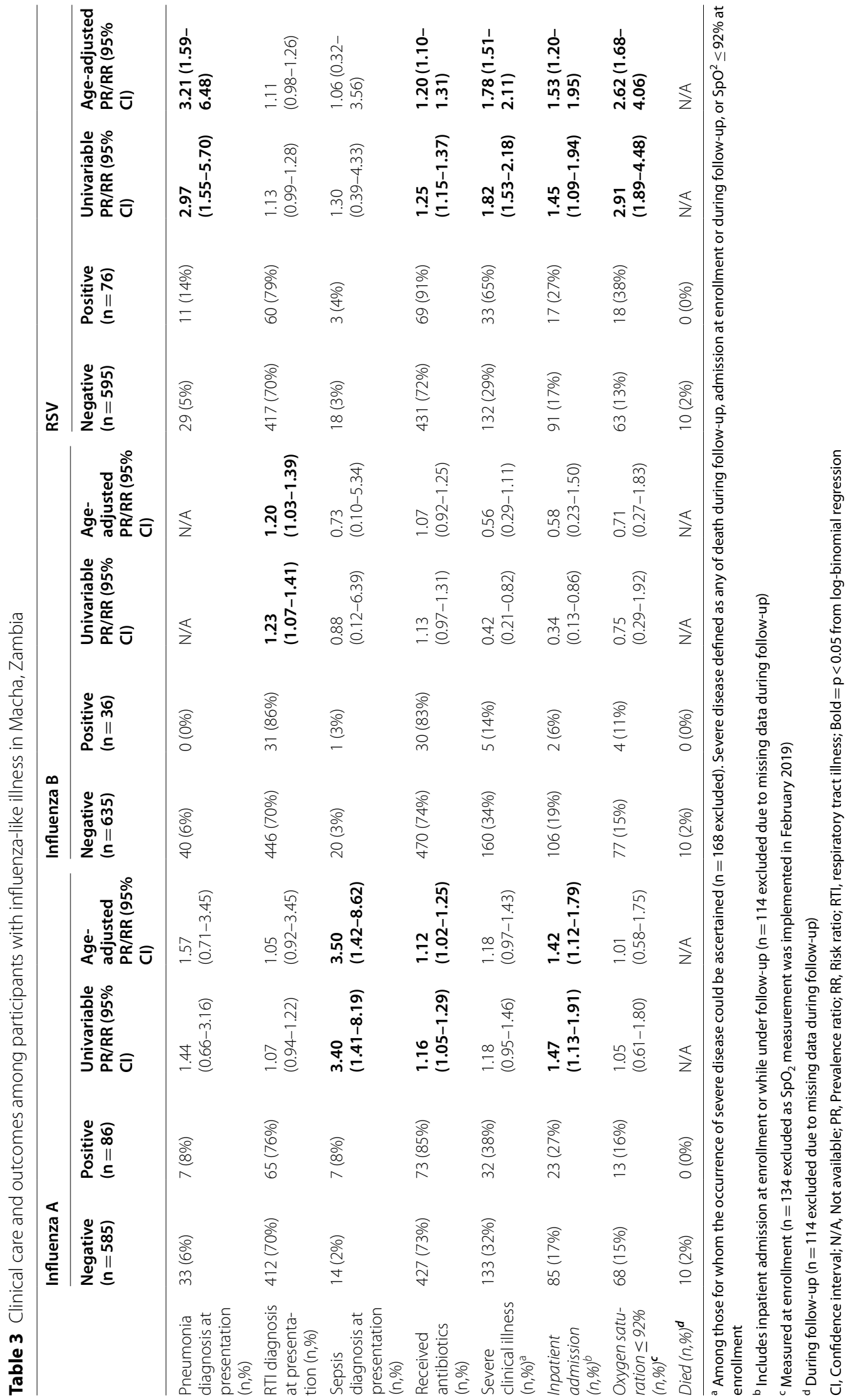


Table 4 Correlates of clinical severity among participants with influenza A, influenza B, and respiratory syncytial virus infection in Macha Zambia

\begin{tabular}{|c|c|c|c|c|c|c|c|c|c|}
\hline & \multicolumn{3}{|c|}{ Influenza A } & \multicolumn{3}{|c|}{ Influenza B } & \multicolumn{3}{|l|}{ RSV } \\
\hline & $\begin{array}{l}\text { Severe } \\
(n=32)\end{array}$ & $\begin{array}{l}\text { Univariable } \\
\text { RR for } \\
\text { severity } \\
\text { (95\% CI) }\end{array}$ & $\begin{array}{l}\text { Age- } \\
\text { adjusted RR } \\
\text { for severity } \\
(95 \% \mathrm{Cl})\end{array}$ & $\begin{array}{l}\text { Severe } \\
(n=5)\end{array}$ & $\begin{array}{l}\text { Univariable } \\
\text { RR for } \\
\text { severity } \\
(95 \% \mathrm{Cl})\end{array}$ & $\begin{array}{l}\text { Age- } \\
\text { adjusted RR } \\
\text { for severity } \\
(95 \% \mathrm{Cl})\end{array}$ & $\begin{array}{l}\text { Severe } \\
(n=33)\end{array}$ & $\begin{array}{l}\text { Univariable } \\
\text { RR for } \\
\text { severity } \\
(95 \% \mathrm{Cl})\end{array}$ & $\begin{array}{l}\text { Age- } \\
\text { adjusted RR } \\
\text { for severity } \\
(95 \% \mathrm{Cl})\end{array}$ \\
\hline \multicolumn{10}{|l|}{ Age, years } \\
\hline $\begin{array}{l}\text { Per year } \\
\text { increase }\end{array}$ & & $\begin{array}{l}1.00 \\
(0.98-1.01)\end{array}$ & - & & $\begin{array}{l}1.00 \\
(0.91-1.09)\end{array}$ & - & & $\begin{array}{l}0.98 \\
(0.93-1.04)\end{array}$ & - \\
\hline $0-5$ months & $3(43 \%)$ & Ref & - & $0(0 \%)$ & $\operatorname{Ref}^{a}$ & - & $8(80 \%)$ & Ref & - \\
\hline 6-11 months & $2(33 \%)$ & $\begin{array}{l}0.78 \\
(0.19-3.21)\end{array}$ & - & $0(0 \%)$ & & - & $9(82 \%)$ & $\begin{array}{l}1.02 \\
(0.67-1.55)\end{array}$ & - \\
\hline $1-4$ & 17 (49\%) & $\begin{array}{l}1.13 \\
(0.45-2.85)\end{array}$ & - & $2(17 \%)$ & & - & 15 (68\%) & $\begin{array}{l}0.85 \\
(0.56-1.30)\end{array}$ & - \\
\hline $5-15$ & $4(29 \%)$ & $\begin{array}{l}0.67 \\
(0.20-2.19)\end{array}$ & - & $2(17 \%)$ & $\begin{array}{l}1.42 \\
(0.23-8.70)\end{array}$ & - & $0(0 \%)$ & N/A & - \\
\hline $16-50$ & $2(22 \%)$ & $\begin{array}{l}0.52 \\
(0.12-2.30)\end{array}$ & - & $1(25 \%)$ & $\begin{array}{l}2.12 \\
(0.25-18.05)\end{array}$ & - & $0(0 \%)$ & N/A & - \\
\hline $51+$ & $4(50 \%)$ & $\begin{array}{l}1.17 \\
(0.39-3.51)\end{array}$ & - & 0 & N/A & - & 1 (100\%) & N/A & - \\
\hline \multicolumn{10}{|l|}{ Gender } \\
\hline Male & 19 (46\%) & Ref & Ref & $0(0 \%)$ & Ref & Ref & 17 (63\%) & Ref & Ref \\
\hline Female & $13(34 \%)$ & $\begin{array}{l}0.74 \\
(0.43-1.28)\end{array}$ & $\begin{array}{l}0.67 \\
(0.39-1.16)\end{array}$ & $5(23 \%)$ & N/A & $\mathrm{N} / \mathrm{A}$ & $16(70 \%)$ & $\begin{array}{l}1.10 \\
(0.74-1.64)\end{array}$ & $\begin{array}{l}1.00 \\
(0.72-1.40)\end{array}$ \\
\hline \multicolumn{10}{|c|}{ Current smoker or smoker in the household $(n, \%)$} \\
\hline No & $27(43 \%)$ & Ref & Ref & $3(10 \%)$ & Ref & Ref & $28(70 \%)$ & Ref & Ref \\
\hline Yes & $5(31 \%)$ & $\begin{array}{l}0.73 \\
(0.33-1.59)\end{array}$ & $\begin{array}{l}0.72 \\
(0.33-1.58)\end{array}$ & $2(100 \%)$ & N/A & N/A & $5(50 \%)$ & $\begin{array}{l}0.71 \\
(0.37-1.37)\end{array}$ & $\begin{array}{l}0.96 \\
(0.60-1.55)\end{array}$ \\
\hline \multicolumn{10}{|c|}{ Underweight $(n, \%)$} \\
\hline No & $21(32 \%)$ & Ref & Ref & $5(17 \%)$ & Ref & Ref & $29(63 \%)$ & Ref & Ref \\
\hline Yes & $10(77 \%)$ & $\begin{array}{l}2.38 \\
(1.50-3.78)\end{array}$ & $\begin{array}{l}2.49 \\
(1.57-3.93)\end{array}$ & $0(0 \%)$ & N/A & N/A & $3(100 \%)$ & N/A & N/A \\
\hline \multicolumn{10}{|c|}{ HIV-infected $(n, \%)$} \\
\hline No & $30(39 \%)$ & Ref & Ref & $5(15 \%)$ & Ref & Ref & $33(66 \%)$ & Ref & Ref \\
\hline Yes & $2(67 \%)$ & $\begin{array}{l}1.69 \\
(0.72-3.94)\end{array}$ & $\begin{array}{l}1.82 \\
(0.55-6.05)\end{array}$ & $0(0 \%)$ & N/A & N/A & $0(0 \%)$ & N/A & N/A \\
\hline \multicolumn{10}{|c|}{ History of tuberculosis $(n, \%)$} \\
\hline No & $31(40 \%)$ & Ref & Ref & $5(15 \%)$ & Ref & Ref & $33(66 \%)$ & Ref & Ref \\
\hline Yes & $1(100 \%)$ & N/A & N/A & $0(0 \%)$ & N/A & $\mathrm{N} / \mathrm{A}$ & $0(0 \%)$ & N/A & N/A \\
\hline \multicolumn{10}{|l|}{ Anemia $(n, \%)$} \\
\hline No & $31(40 \%)$ & Ref & Ref & $5(15 \%)$ & Ref & Ref & $31(65 \%)$ & Ref & Ref \\
\hline Yes & $1(50 \%)$ & $\begin{array}{l}1.24 \\
(0.30-5.10)\end{array}$ & $\begin{array}{l}1.00 \\
(0.20-4.95)\end{array}$ & $0(0 \%)$ & N/A & $\mathrm{N} / \mathrm{A}$ & $2(100 \%)$ & N/A & N/A \\
\hline \multicolumn{10}{|c|}{ Objective fever $(n, \%)$} \\
\hline No & $22(39 \%)$ & Ref & Ref & $3(15 \%)$ & Ref & Ref & $28(65 \%)$ & Ref & Ref \\
\hline Yes & $10(43 \%)$ & $\begin{array}{l}1.11 \\
(0.63-1.95)\end{array}$ & $\begin{array}{l}1.14 \\
(0.66-1.98)\end{array}$ & $2(15 \%)$ & $\begin{array}{l}1.03 \\
(0.20-5.33)\end{array}$ & $\begin{array}{l}0.95 \\
(0.17-5.30)\end{array}$ & $5(71 \%)$ & $\begin{array}{l}1.10 \\
(0.65-1.84)\end{array}$ & $\begin{array}{l}1.18 \\
(0.79-1.76)\end{array}$ \\
\hline \multicolumn{10}{|l|}{ Diarrhea $(n, \%)$} \\
\hline No & $28(44 \%)$ & Ref & Ref & $2(8 \%)$ & Ref & Ref & $29(67 \%)$ & Ref & Ref \\
\hline Yes & $4(27 \%)$ & $\begin{array}{l}0.60 \\
(0.25-1.45)\end{array}$ & $\begin{array}{l}0.54 \\
(0.22-1.31)\end{array}$ & $3(38 \%)$ & $\begin{array}{l}4.69 \\
(0.94-23.27)\end{array}$ & $\begin{array}{l}4.65 \\
(0.88-24.42)\end{array}$ & $4(57 \%)$ & $\begin{array}{l}0.85 \\
(0.43-1.66)\end{array}$ & $\begin{array}{l}0.69 \\
(0.34-1.40)\end{array}$ \\
\hline \multicolumn{10}{|c|}{ Duration of ILI symptoms at presentation, days } \\
\hline $\begin{array}{l}\text { Per day } \\
\text { increase }\end{array}$ & & $\begin{array}{l}1.05 \\
(0.89-1.23)\end{array}$ & $\begin{array}{l}1.05 \\
(0.91-1.22)\end{array}$ & & $\begin{array}{l}0.81 \\
(0.48-1.39)\end{array}$ & $\begin{array}{l}0.76 \\
(0.42-1.36)\end{array}$ & & $\begin{array}{l}1.06 \\
(0.93-1.21)\end{array}$ & $\begin{array}{l}1.07 \\
(0.93-1.23)\end{array}$ \\
\hline $1-2$ & $5(38 \%)$ & Ref & Ref & $2(25 \%)$ & Ref & Ref & $4(50 \%)$ & Ref & Ref \\
\hline
\end{tabular}


Table 4 (continued)

\begin{tabular}{|c|c|c|c|c|c|c|c|c|c|}
\hline & Influenza & & & Influenz & & & RSV & & \\
\hline & $\begin{array}{l}\text { Severe } \\
(n=32)\end{array}$ & $\begin{array}{l}\text { Univariable } \\
\text { RR for } \\
\text { severity } \\
(95 \% \mathrm{Cl})\end{array}$ & $\begin{array}{l}\text { Age- } \\
\text { adjusted RR } \\
\text { for severity } \\
(95 \% \mathrm{Cl})\end{array}$ & $\begin{array}{l}\text { Severe } \\
(n=5)\end{array}$ & $\begin{array}{l}\text { Univariable } \\
\text { RR for } \\
\text { severity } \\
(95 \% \mathrm{Cl})\end{array}$ & $\begin{array}{l}\text { Age- } \\
\text { adjusted RR } \\
\text { for severity } \\
(95 \% \mathrm{Cl})\end{array}$ & $\begin{array}{l}\text { Severe } \\
(n=33)\end{array}$ & $\begin{array}{l}\text { Univariable } \\
\text { RR for } \\
\text { severity } \\
(95 \% \mathrm{Cl})\end{array}$ & $\begin{array}{l}\text { Age- } \\
\text { adjusted RR } \\
\text { for severity } \\
(95 \% \mathrm{Cl})\end{array}$ \\
\hline $3-5$ & 24 (42\%) & $\begin{array}{l}1.09 \\
(0.52-2.32)\end{array}$ & $\begin{array}{l}1.27 \\
(0.60-2.71)\end{array}$ & $3(15 \%)$ & $\begin{array}{l}0.60 \\
(0.12-2.94)\end{array}$ & $\begin{array}{l}0.41 \\
(0.07-2.52)\end{array}$ & $23(68 \%)$ & $\begin{array}{l}1.35 \\
(0.65-2.81)\end{array}$ & $\begin{array}{l}1.31 \\
(0.68-2.54)\end{array}$ \\
\hline $6+$ & $3(33 \%)$ & $\begin{array}{l}0.87 \\
(0.27-2.74)\end{array}$ & $\begin{array}{l}0.44 \\
(0.06-3.21)\end{array}$ & $0(0 \%)$ & N/A & N/A & $6(75 \%)$ & $\begin{array}{l}1.50 \\
(0.67-3.34)\end{array}$ & $\begin{array}{l}1.64 \\
(0.77-3.48)\end{array}$ \\
\hline
\end{tabular}

${ }^{a}$ Ages $0-4$ years is designated as the reference to enable comparisons across age groups

$\mathrm{Cl}$, Confidence interval; ILI, influenza-like illness; N/A, Not available (distribution not significantly different based on Fisher's exact testing); REF, Reference group; RR, Risk ratio; $\mathrm{RTI}$, respiratory tract infection; $B$ old $=\mathrm{p}<0.05$ from log-binomial regression

site more than one in six outpatients presented with ILI and we found a sizeable annual prevalence of both influenza viruses (18\%) and RSV (11\%) among symptomatic outpatients across age groups. RSV in particular was associated with clinically severe respiratory disease. Overall, $75 \%$ of patients presenting with ILI symptoms were prescribed empiric antibiotics, including $87 \%$ of those with a viral infection. These findings highlight the burden of influenza viruses and RSV in rural Zambia and the importance of context-specific epidemiologic and etiologic knowledge for clinicians and public health practitioners.

While the study period for the present analysis occurred prior to the start of the COVID-19 pandemic, the research infrastructure and testing platforms described are well-suited, moving forward, to aid in furthering our understanding of influenza viruses and RSV during the current and future outbreaks. Recent reports from the region, including Zambia, highlight the growing concurrent increase in both severe acute respiratory syndrome coronavirus 2 (SARS-CoV-2) infections as well as influenza virus infections $[18,29]$. Additionally, the descriptions of clinical course and risk factors for severe disease may be of use to both local clinicians and public health practitioners in treatment and planning functions.

The observed prevalence of influenza virus infection in this study was markedly higher than that reported in published studies from Lusaka where prevalence among outpatients with ILI ranged from $3.7 \%$ [30] to $12.6 \%$ [4, $15,16,30,31]$. These differences may be due to several factors, including differences in the assays used and the age distribution of the study participants. In the study by Mizuta et al. [30], for example, over $43 \%$ were children under 1 year, an age group with among the lowest influenza prevalence in our study. However, these differences may also suggest an increased burden of symptomatic respiratory infections in rural areas. Our work complements emerging data from the Zambian Ministry of Health-led surveillance in urban centers suggesting that 2019 may have been a year of particularly intense influenza activity [18].

In this rural setting, we found a distinct but prolonged period of influenza virus activity with cases detected from April to November. These findings contrast both with published estimates of seasonality in Zambia, which suggest a single prevalence peak during the cold, dry season (May-August), [15, 30], and with multi-year data from ongoing surveillance efforts from urban clinical care centers in Lusaka and Ndola that suggest nearly year-round transmission with multiple annual peaks [18]. With regard to RSV, prior studies from Lusaka $[15,17$, 32] and other southern African countries [33, 34] report prolonged RSV seasons and multiple annual peaks in RSV incidence, along with temporal coincidence of RSV and influenza virus activity. However, in this study these infections had distinct peaks and were separately responsible for driving a bimodal temporal distribution of respiratory cases in the outpatient population.

Our study period coincided with a period of significant drought in southern Zambia. Given notable associations of respiratory virus transmission with precipitation and humidity $[35,36]$, this may have impacted the observed temporal patterns of viral prevalence and could explain, at least in part, the differences observed between our study site and northerly urban areas that experienced more rainfall. Troublingly, drought conditions exacerbated existing food insecurity and undernutrition in the region [37]. Our findings of an association between underweight with both influenza A virus infection and more severe clinical influenza illness complement evidence from animal studies and observations among human patients in South Africa [38-40].

This study was among the first to pilot rapid point-ofcare viral testing in southern Zambia, an area with little 
clinical laboratory infrastructure. The Cepheid GeneXpert platform has been broadly adopted in sub-Saharan Africa for use in tuberculosis diagnosis, is increasingly being used for early infant diagnosis and quantification of HIV viral load [41-43], and has most recently been used for rapid diagnosis of SARS-CoV-2 during the current pandemic. Broadening the assay's scope of use could be key in facilitating sensitive and reliable microbiological testing even in remote, low-resource environments.

This study is not without limitations. First, given the exploratory nature of this study and its relatively small sample size it is difficult to draw causal conclusions on factors associated with clinical disease and severity. Further study in this and other similar settings will be needed to bolster these investigations. Second, as the primary focus of the surveillance platform was influenza virus, a case definition based on ILI was used to identify potential participants. Thus, while our findings may be useful for informing clinical diagnosis of viral infection, we could not detect asymptomatic or pauci-symptomatic infections. In addition, the requirement for fever in the case definition may have led to an underestimation of the burden of symptomatic RSV as a substantial proportion of RSV-infected young children and elderly patients present without fever [44]. Third, 25\% of participants were excluded from the assessment of severe clinical illness due to missing data. As retention may be related to poor health outcomes, this may have led to an underestimation of the proportion experiencing severe clinical illness. However, as over half of excluded participants were only missing data on $\mathrm{SpO}^{2}$ due to the inclusion of this measure after the study was initiated, this is unlikely to have biased the risk factor analysis. Finally, the present study reports on a single year of viral surveillance. More complete understanding of seasonality and disease patterns will require sustained efforts across multiple years.

In summary, rural southern Zambia bears a large burden of influenza- and RSV- related disease. Rural areas that are home to the majority of national and regional populations have distinct features and risk factors compared to urban centers yet have been consistently under-surveilled to date. Our findings point to important in-country heterogeneity in the prevalence and epidemiology of respiratory infections and highlight the importance of continued, wide-ranging surveillance efforts in capturing the true burden of disease. Such surveillance programs that can provide more granular, context-specific information are key to planning for and responding to existing and emerging disease threats.

\section{Abbreviations}

$\% \mathrm{SpO}_{2}$ : Peripheral capillary oxygen saturation; adjPR: Age-adjusted prevalence ratio; adjRR: Age-adjusted risk ratio; ARI: Acute respiratory infection; BMI: Body mass index; Cl: Confidence interval; ILI: Influenza-like illness; N/A: Not available; OPD: Outpatient department; PR: Prevalence ratio; RR: Risk ratio; RSV: Respiratory syncytial virus; RTI: Respiratory tract infection.

\section{Supplementary Information}

The online version contains supplementary material available at https://doi. org/10.1186/s12879-021-06677-5.

Additional file 1: Sampling scheme for participants with influenza-like illness recruited from the outpatient department at Macha Hospital

Additional file 2: Symptomatology and clinical presentation of influenza virus and RSV infections by age

Additional file 3: Age-specific seasonal trends over time in A) influenzalike illness, B) influenza A virus, C) influenza B virus, and D) respiratory syncytial virus prevalence among outpatients in Macha, Zambia, December 2018 to December 2019

Additional file 4: Age-distribution of all outpatients, outpatients with influenza-like illness, and outpatient study participants

Additional file 5: Age-distribution of all inpatients, inpatients with acute respiratory illness, and inpatient study participants

Additional file 6: Temperature, precipitation, and viral infection in Macha Zambia, December 2018 to December 2019.

\section{Acknowledgements}

The authors would like to acknowledge Morris Sianyinda, Trinity Sibalwa, Caren Mweene, Yvonne Phiri, Calton Mwendo, Passwell Munachoonga, and Mathias Muleka for their help in data collection. The authors would also like to thank Justin Hardick, Thomas Mehoke, Petere Thielen, and Jared Evans for their guidance and insights.

\section{Authors' contributions}

GL, CS, PT, RR, and AP conceptualized the study and study protocols. GL and CS carried out all analyses. GL wrote the manuscript with input from all authors. PS coordinated data collection. MH coordinated laboratory testing. $\mathrm{KS}, \mathrm{KF}, \mathrm{MM}$ and $\mathrm{ES}$ provided regular input on study direction, protocols, and planning. All authors read and approved the final manuscript.

\section{Funding}

The work was supported by the National Institute of Allergy and Infectious Diseases (contract HHSN272201400007C) awarded to the Johns Hopkins Center of Excellence in Influenza Research and Surveillance (JHCEIRS). Any opinions, findings, conclusions, or recommendations expressed are those of the authors and do not represent the policy or position of NIAID or NIH. The funding agency had no role in the analysis of the data, writing of the manuscript, or the decision to submit the manuscript for publication.

\section{Availability of data and materials}

Under the Research Health Act, the Government of Zambia does not allow public access to data collected in Zambia. All investigators interested in the data are required to submit a written request to the Ministry of Health. Contact Dr. Catherine Sutcliffe (csutcli1@jhu.edu) to coordinate the request.

\section{Declarations}

\section{Ethical approval and consent to participate}

In accordance with relevant guidelines and regulations, all study protocols and materials were approved by the Johns Hopkins School of Medicine Institutional Review Board, the Macha Research Trust Institutional Review Board, and the Zambian National Health Research Authority. Adult participants and parents or legal guardians of child participants provided written informed consent. In addition, children 12-15 years of age provided written assent. 


\section{Consent for publication \\ Not applicable.}

\section{Competing interests}

The authors declare that they have no known competing financial interests or personal relationships that might have influenced the work reported in this publication.

\begin{abstract}
Author details
${ }^{1}$ Department of Epidemiology, Johns Hopkins University Bloomberg School of Public Health, 615 N. Wolfe Street, Room E6535, Baltimore, MD 21205, USA ${ }^{2}$ Johns Hopkins University School of Medicine, Baltimore, MD, USA. ${ }^{3}$ Macha Research Trust, Macha, Choma, Zambia. ${ }^{4}$ Department of Emergency Medicine, Johns Hopkins University School of Medicine, Baltimore, MD, USA. ${ }^{5}$ Department of Microbiology and Immunology, Johns Hopkins University Bloomberg School of Public Health, Baltimore, MD, USA. ${ }^{6}$ Virology Laboratory, University Teaching Hospital, Lusaka, Zambia.
\end{abstract}

Received: 17 May 2021 Accepted: 10 September 2021 Published online: 21 September 2021

\section{References}

1. Shi T, McAllister DA, O'Brien KL, Simoes EAF, Madhi SA, Gessner BD, Polack FP, Balsells E, Acacio S, Aguayo C, et al. Global, regional, and national disease burden estimates of acute lower respiratory infections due to respiratory syncytial virus in young children in 2015: a systematic review and modelling study. Lancet. 2017;390(10098):946-58.

2. Iuliano AD, Roguski KM, Chang HH, Muscatello DJ, Palekar R, Tempia S, Cohen C, Gran JM, Schanzer D, Cowling BJ, et al. Estimates of global seasonal influenza-associated respiratory mortality: a modelling study. Lancet. 2018;391(10127):1285-300.

3. Katz MA, Schoub BD, Heraud JM, Breiman RF, Njenga MK, Widdowson MA. Influenza in Africa: uncovering the epidemiology of a long-overlooked disease. J Infect Dis. 2012;206(Suppl 1):S1-4.

4. PERCH Study Group. Causes of severe pneumonia requiring hospital admission in children without HIV infection from Africa and Asia: the PERCH multi-country case-control study. Lancet. 2019;394(10200):757-79.

5. Stein RT, Bont LJ, Zar H, Polack FP, Park C, Claxton A, Borok G, Butylkova Y, Wegzyn C. Respiratory syncytial virus hospitalization and mortality: systematic review and meta-analysis. Pediatr Pulmonol. 2017;52(4):556-69.

6. Cohen C, Simonsen L, Kang JW, Miller M, McAnerney J, Blumberg $L$, Schoub B, Madhi SA, Viboud C. Elevated influenza-related excess mortality in South African elderly individuals, 1998-2005. Clin Infect Dis. 2010;51(12):1362-9.

7. Paynter S, Ware RS, Lucero MG, Tallo V, Nohynek H, Weinstein P, Williams G, Sly PD, Simões EA. Malnutrition: a risk factor for severe respiratory syncytial virus infection and hospitalization. Pediatr Infect Dis J. 2014;33(3):267-71.

8. Ho A, Aston SJ, Jary H, Mitchell T, Alaerts M, Menyere M, Mallewa J, Nyirenda M, Everett D, Heyderman RS, et al. Impact of HIV on the burden and severity of influenza illness in Malawian adults: a prospective cohort and parallel case-control study. Clin Infect Dis. 2017;66(6):865-76.

9. Walaza S, Tempia S, Dawood H, Variava E, Wolter N, Dreyer A, Moyes J, Von Mollendorf C, McMorrow M, Von Gottberg A, et al. The impact of influenza and tuberculosis interaction on mortality among individuals aged $\geq 15$ years hospitalized with severe respiratory illness in South Africa, 2010-2016. Open Forum Infect Dis. 2019;6(3):ofz020.

10. The World Bank. World Bank Open Data: Rural population. https://data. worldbank.org/indicator/SP.RUR.TOTL. Accessed 19 Aug 2021.

11. Beegle K, Christiaensen L, Dabalen A, Gaddis I. Poverty in a rising Africa. Africa Poverty Report. Washington, DC: World Bank; 2016.

12. Sikalengo G, Hella J, Mhimbira F, Rutaihwa LK, Bani F, Ndege R, Sasamalo M, Kamwela L, Said K, Mhalu G, et al. Distinct clinical characteristics and helminth co-infections in adult tuberculosis patients from urban compared to rural Tanzania. Infect Dis Poverty. 2018;7(1):24.

13. Magadi MA. Understanding the urban-rural disparity in HIV and poverty nexus: the case of Kenya. J Public Health (Oxf). 2017:39(3):e63-72
14. Romay-Barja M, Jarrin I, Ncogo P, Nseng G, Sagrado MJ, Santana-Morales MA, Aparicio P, Aparcio P, Valladares B, Riloha M, et al. Rural-urban differences in household treatment-seeking behaviour for suspected malaria in children at Bata District, Equatorial Guinea. PLoS One. 2015;10(8):e0135887.

15. Theo A, Liwewe M, Ndumba I, Mupila Z, Tambatamba B, Mutemba C, Somwe SW, Mwinga A, Tempia S, Monze M. Influenza surveillance in Zambia, 2008-2009. J Infect Dis. 2012;206(Suppl 1):S173-177.

16. Theo A, Tempia S, Cohen AL, Simusika P, Chentulo E, Chikamukwa CM, Monze M. The national burden of influenza-associated severe acute respiratory illness hospitalization in Zambia, 2011-2014. Influenza Other Respir Viruses. 2018;12(1):46-53.

17. Simusika P, Bateman AC, Theo A, Kwenda G, Mfula C, Chentulo E, Monze M. Identification of viral and bacterial pathogens from hospitalized children with severe acute respiratory illness in Lusaka, Zambia, 2011-2012: a cross-sectional study. BMC Infect Dis. 2015;15:52.

18. WHO. FluNet. https://www.who.int/influenza/gisrs_laboratory/flunet/en/ Accessed 19 Aug 2021.

19. Sutcliffe CG, Kobayashi T, Hamapumbu H, Shields T, Mharakurwa S, Thuma PE, Louis TA, Glass G, Moss WJ. Reduced risk of malaria parasitemia following household screening and treatment: a cross-sectional and longitudinal cohort study. PLoS One. 2012;7(2):e31396.

20. Ippolito MM, Searle KM, Hamapumbu H, Shields TM, Stevenson JC, Thuma PE, Moss WJ, for the Southern Africa International Center of Excellence for Malaria Research. House structure is associated with Plasmodium falciparum infection in a low-transmission setting in southern Zambia. Am J Trop Med Hyg. 2017;97(5):1561-7.

21. Ministry of Health (MOH) (Zambia), Central Statistical Office (CSO) (Zambia]), ICF: Zambia demographic and health survey 2018: key indicators. Rockville, Maryland; 2019: 25.

22. CDC. U.S. Influenza Surveillance System: purpose and methods. https:// www.cdc.gov/flu/weekly/overview.htm. Accessed 12 July 12021.

23. Deitchler M, Ballard T, Swindale A, Coates J. Validation of a measure of household hunger for cross-cultural use. Food and nutrition technical assistance. Washington, DC: USAID; 2010.

24. Banerjee D, Kanwar N, Hassan F, Essmyer C, Selvarangan R. Comparison of six sample-to-answer influenza $A / B$ and respiratory syncytial virus nucleic acid amplification assays using respiratory specimens from children. $J$ Clin Microbiol. 2018;56(11):e00930-18. https://doi.org/10.1128/JCM. 00930-18.

25. WHO Multicentre Growth Reference Study Group. WHO child growth standards: methods and development: Length/height-for-age, weightfor-age, weight-for-length, weight-for-height and body mass index-forage. Geneva: World Health Organization; 2006.

26. WHO. Integrated Management of Childhood IIIness for High HIV Settings. Geneva, Switzerland: World Health Organization; 2008.

27. Majumdar SR, Eurich DT, Gamble JM, Senthilselvan A, Marrie TJ. Oxygen saturations less than $92 \%$ are associated with major adverse events in outpatients with pneumonia: a population-based cohort study. Clin Infect Dis. 2011;52(3):325-31.

28. McCollum ED, Ginsburg AS. Outpatient management of children with World Health Organization chest indrawing pneumonia: implementation risks and proposed solutions. Clin Infect Dis. 2017;65(9):1560-4.

29. Dong E, Du H, Gardner L. An interactive web-based dashboard to track COVID-19 in real time. Lancet Infect Dis. 2020;20(5):533-4.

30. Mizuta K, Oshitani H, Saijo M, Mpabalwani EM, Kasolo FC, Luo NP, Suzuki $H$, Numazaki Y. Epidemiology of influenza virus infections in children with acute respiratory infections in Zambia. Ann Trop Paediatr. 1997;17(2):115-9.

31. Simusika P, Tempia S, Chentulo E, Polansky L, Mazaba ML, Ndumba I, Mbewe QK, Monze M. An evaluation of the Zambia influenza sentinel surveillance system, 2011-2017. BMC Health Serv Res. 2020;20(1):35.

32. Thea D, Prosperi C, Gill C. PERCH study results - Influenza- and RSVpositivity from children with severe pneumonia in Lusaka, Zambia (unpublished); 2019.

33. Madhi SA, Cutland CL, Downs S, Jones S, van Niekerk N, Simoes EA, Nunes MC. Burden of respiratory syncytial virus infection in South African human immunodeficiency virus (HIV)-infected and HIV-uninfected pregnant and postpartum women: a longitudinal cohort study. Clin Infect Dis. 2018;66(11):1658-65 
34. Peterson I, Bar-Zeev N, Kennedy N, Ho A, Newberry L, SanJoaquin MA, Menyere M, Alaerts M, Mapurisa G, Chilombe M, et al. Respiratory virus-associated severe acute respiratory illness and viral clustering in Malawian children in a setting with a high prevalence of HIV infection, malaria, and malnutrition. J Infect Dis. 2016;214(11):1700-11.

35. Yang W, Cummings MJ, Bakamutumaho B, Kayiwa J, Owor N, Namagambo B, Byaruhanga T, Lutwama JJ, O'Donnell MR, Shaman J. Dynamics of influenza in tropical Africa: temperature, humidity, and cocirculating (sub)types. Influenza Other Respir Viruses. 2018:12(4):446-56.

36. Baker RE, Mahmud AS, Wagner CE, Yang W, Pitzer VE, Viboud C, Vecchi GA, Metcalf CJE, Grenfell BT. Epidemic dynamics of respiratory syncytial virus in current and future climates. Nat Commun. 2019;10(1):5512.

37. United Nations Office for the Coordination of Humanitarian Affairs (OCHA). Zambia: Prolonged drought increases food insecurity. https:// www.unocha.org/story/zambia-prolonged-drought-increases-food-insec urity. Accessed 19 August 2021.

38. Taylor AK, Cao W, Vora KP, De La Cruz J, Shieh WJ, Zaki SR, Katz JM, Sambhara S, Gangappa S. Protein energy malnutrition decreases immunity and increases susceptibility to influenza infection in mice. J Infect Dis. 2013;207(3):501-10

39. Ritz BW, Gardner EM. Malnutrition and energy restriction differentially affect viral immunity. J Nutr. 2006;136(5):1141-4.

40. Tempia S, Walaza S, Moyes J, Cohen AL, von Mollendorf C, Treurnicht FK, Venter M, Pretorius M, Hellferscee O, Mtshali S, et al. Risk factors for influenza-associated severe acute respiratory illness hospitalization in South Africa, 2012-2015. Open Forum Infect Dis. 2017;4(1):ofw262.

41. WHO. Roadmap for rolling out Xpert MTB/RIF for rapid diagnosis of TB and MDR-TB. Geneva, Switzerland: World Health Organization; 2010.

42. Moyo S, Mohammed T, Wirth KE, Prague M, Bennett K, Holme MP, Mupfumi L, Sebogodi P, Moraka NO, Boleo C, et al. Point-of-care Cepheid Xpert HIV-1 viral load test in rural african communities is feasible and reliable. J Clin Microbiol. 2016;54(12):3050-5.

43. Bianchi F, Cohn J, Sacks E, Bailey R, Lemaire JF, Machekano R, on behalf of the EGPAF POC EID Study Team. Evaluation of a routine point-of-care intervention for early infant diagnosis of HIV: an observational study in eight African countries. Lancet HIV. 2019;6(6):e373-81.

44. Hirve S, Crawford N, Palekar R, Zhang W, WHO RSV Surveillance Group. Clinical characteristics, predictors, and performance of case definition - Interim results from the WHO global respiratory syncytial virus surveillance pilot. Influenza Other Respir Viruses. 2019;14(6):647-57.

\section{Publisher's Note}

Springer Nature remains neutral with regard to jurisdictional claims in published maps and institutional affiliations.
Ready to submit your research? Choose BMC and benefit from:

- fast, convenient online submission

- thorough peer review by experienced researchers in your field

- rapid publication on acceptance

- support for research data, including large and complex data types

- gold Open Access which fosters wider collaboration and increased citations

- maximum visibility for your research: over 100M website views per year

At BMC, research is always in progress.

Learn more biomedcentral.com/submissions 